\title{
RNA localization along the anteroposterior axis of the Drosophila oocyte requires PKA-mediated signal transduction to direct normal microtubule organization
}

\author{
Mary Ellen Lane ${ }^{1}$ and Daniel Kalderon \\ Department of Biological Sciences, Columbia University, New York, New York 10027 USA
}

\begin{abstract}
Microtubule polarity has been implicated as the basis for polarized localization of morphogenetic determinants that specify the anteroposterior axis in Drosophila oocytes. We describe mutations affecting Protein Kinase A (PKA) that act in the germ line to disrupt both microtubule distribution and RNA localization along this axis. In normal oocytes, the site of microtubule nucleation shifts from posterior to anterior immediately prior to polarized localization of bicoid and oskar RNAs. In PKA-deficient oocytes, posterior microtubules are present during this transition, oskar RNA fails to accumulate at the posterior, and bicoid RNA accumulates at both ends of the oocyte. Similar RNA mislocalization patterns previously reported for Notch and Delta mutants suggest that PKA transduces a signal for microtubule reorganization that is sent by posteriorly located follicle cells.
\end{abstract}

[Key Words: Drosophila; oogenesis; protein kinase A; RNA localization; microtubules]

Received September 16, 1994; revised version accepted October 26, 1994.

Localization of positional determinants is an important strategy for the specification of cell fate in early embryonic development. It is often achieved by localizing mRNAs in the developing oocyte (Macdonald 1992). The mechanisms responsible for localizing RNAs to distinct regions of a cell are poorly understood but have been shown in several cases to depend on specific sequences at the 3' end of the RNA and an intact microtubule cytoskeleton /Yisraeli et al. 1990; Pokrywka and Stephenson 1991; Mowry and Melton 1992; Kim-Ha et al. 1993; Macdonald et al. 1993). By analogy to organelle movement within axons, it is generally thought that motor proteins of the kinesin and dynein families mediate the transport of RNAs along microtubules (Ainger et al. 1993; Hirokawa 1993; Willhelm and Vale 1993). Because motor proteins recognize the polarity of microtubules the generation of a microtubule cytoskeleton of appropriate polarity is an essential prerequisite for directed RNA transport by this mechanism.

In Drosophila embryos, specification of cell fates along the anteroposterior axis has been shown to require a gradient of bicoid protein, which is achieved by the diffusion of protein translated from mRNA localized at the

${ }^{1}$ Present address: Friedrich-Miescher Labor der Max Plank Gesellschaft, Tübingen, 72076 Germany. anterior pole of the embryo. Similarly, specification of germ cells and localization of the posterior determinant, nanos, have been shown to require posterior localization of oskar (osk) mRNA (for review, see Ferrandon and St. Johnston 1992). Localization of bicoid $(b c d)$ and osk RNAs is initiated early in oogenesis as they accumulate in the oocyte of the germarium and early vitellarium. The subsequent accumulation of $b c d$ and osk RNAs at opposite poles of the oocyte (St. Johnston et al. 1989, 1991; Ephrussi et al. 1991; Kim-Ha et al. 1991) occurs at a time when the oocyte is surrounded by specialized follicle cells and is disrupted by drugs that depolymerize microtubules (Pokrywka and Stephenson 1991; Theurkauf et al. 1993).

Direct visualization of microtubule distribution during Drosophila oogenesis suggests how microtubules may act to direct RNA transport (Theurkauf et al. 1992, 1993). The presence of a microtubule organizing center (MTOC) in the oocyte has been demonstrated by partial depolymerization experiments. This MTOC extends microtubules into the nurse cells, which are connected to the oocyte by intercellular bridges, providing a path for the initial accumulation of RNAs in the oocyte, which is itself transcriptionally inactive throughout most of oogenesis. A redistribution of microtubules occurs during mid-oogenesis, immediately prior to the polarized localization of $b c d$ and osk RNAs. This involves the disap- 
pearance of the posteriorly localized MTOC and the nucleation of microtubules at the anterior margin of the oocyte, which subsequently extend to the extreme posterior. Because microtubules are nucleated at the minus end (Mitchison 1992), this provides a microtubule cytoskeleton of uniform anteroposterior polarity that could accommodate transport of $b c d$ RNA to the anterior by minus-end-directed motor proteins, whereas osk RNA moves to the posterior in association with plus-end-directed motor proteins.

A small number of mutations that disrupt bcd or osk RNA localization during oogenesis have been identified on the basis of an embryonic phenotype similar to that of $b c d$ or osk. Thus, mutations in the exuperantia (exu) or swallow (swa) genes result in embryos that lack some anterior structures; these mutations also result in the failure to initiate $(e x u)$ or maintain $(s w a)$ anterior localization of bcd RNA in the developing oocyte ( $\mathrm{St}$. Johnston et al. 1989). Similarly, mutations in cappuccino (capu), spire (spir), or staufen (stau), which result in embryos lacking germ cells and with posterior structures deleted, also cause mislocalization of osk RNA in oocytes (Ephrussi et al. 1991; Kim-Ha et al. 1991; St. Johnston et al. 1991). Because exu, swa, capu, stau, and spir activities are required in the germ cells, most models for the generation of anteroposterior polarity involve a germ-line autonomous mechanism.

Conditional alleles of the neurogenic genes, $\operatorname{Notch}(N)$ and Delta $(D 1)$, can also disnupt the localization of RNAs in the oocyte (Ruohola et al. 1991). Although these mutations have pleiotropic effects on oogenesis, carefully controlled temperature shift experiments indicate that they have a specific role in the determination of oocyte polarity. Furthermore, $N$ and $\mathrm{Dl}$ mutations affect the localization patterns of both bcd and osk, making them unique among genes so far identified. In addition, $N$ and $D l$ functions appear to be required in somatic follicle cells rather than germline cells for normal oocyte polarity.

We now report that germ-line protein kinase A (PKA) mutations cause similar mislocalizations of $b c d$ and osk RNAs to those observed previously to result from $N \mathrm{mu}$ tations. This supports the hypothesis that cell communication between follicle cells and the oocyte is essential for the machinery that localizes RNAs along the anteroposterior axis (Ruohola et al. 1991). We also show that PKA mutations selectively disrupt the organization of microtubules at the posterior of the oocyte at the time of $b c d$ and osk RNA localization. This can account for the observed mislocalization of $b c d$ and osk RNAs at ectopic microtubule minus and plus ends, respectively, and therefore provides key evidence in favor of a direct role for microtubule-based transport in RNA localization. Therefore, we suggest that PKA normally acts in the oocyte in response to a temporal signal from posteriorly located follicle cells to mediate the disassembly of microtubules at the posterior and thereby permit a uniform gradient of microtubule polarity that is essential for the correct localization of RNA molecules along the anteroposterior axis of the oocyte.

\section{Results}

PKA mutations disrupt bed and osk RNA localization

We have previously described female sterile alleles of the catalytic subunit gene, $D C O$, of Drosophila PKA (Lane and Kalderon 1993). Females that carry the deficiency $D f(2 L) T w 2$, which deletes the 3 '-untranslated region of the $D C O$ gene in trans to null alleles do not lay eggs, although mature oocytes are produced. To examine more carefully the extent to which oocyte development is affected by PKA mutations, we stained oocytes with probes against RNAs that become localized to specific regions of the oocyte during oogenesis.

In wild-type ovaries, RNA for the osk gene accumulates in one cell of the developing cyst in the germarium and throughout stages 1-6 of oogenesis (Ephrussi et al. 1991; Kim-Ha et al. 1991). Between stages 8 and 9, osk RNA becomes localized to the posterior of the oocyte, with some of the RNA transiently accumulating at the oocyte anterior early in stage 8 (Fig. lB). By stage 9 , all of the osk RNA in the oocyte is tightly localized to the posterior pole, where it remains detectable until early embryogenesis. This localization has been shown to be required for the subsequent posterior localization of many RNAs and proteins that are required for germ cell specification and abdominal patterning, including vasa protein and nanos RNA (Hay et al. 1990; Lasko and Ashburner 1990; Gavis and Lehmann 1992).

We observed oocyte-specific accumulation of osk RNA in stage 1-6 egg chambers from PKA-deficient females (Fig. 1A). However, in $42 \%(80 / 190)$ of all stage 8 and 9 egg chambers, osk RNA failed to accumulate at the posterior pole and, instead, appeared concentrated in the middle of the oocyte (Fig. 1C). This ectopic localization persisted until stage 10 . After this time we saw only oocytes with normal osk localization and oocytes in which no osk RNA was detectable. Staining PKA mutant egg chambers with antibodies against the vasa protein showed that vasa failed to localize to the posterior in $48 \%(111 / 228)$ of stage 10 egg chambers (data not shown), as would be predicted from the absence of posteriorly localized osk RNA.

RNA for the anterior morphogen bcd also becomes localized in a stepwise manner (St. Johnston et al. 1989), first accumulating in the oocyte during stage 5 , and becoming localized to the anterior margin at stage 8 (Fig. ID), where it remains until early embryogenesis. In $28 \%$ (47/167) of all stage 8 egg chambers from $D f(2 L) T w 2 /$ $D C 0^{B 3}$ females, we saw transient accumulation of $b c d$ RNA at the posterior as well as the anterior end (Fig. lE). The anterior localization appeared indistinguishable from wild type and persisted throughout oogenesis. By mid-stage $9, b c d$ was no longer detected at the oocyte posterior.

Specification of the dorsoventral axis can also be detected by RNA localization patterns in the oocyte. Neuman-Silberberg and Schüpbach (1993) have shown that RNA for the gurken (grk) gene, which is involved in the communication of positional information between the oocyte and the surrounding follicle cells, is localized first 
Figure 1. PKA mutations disrupt $b c d$ and osk RNA localization. In situ hybridization for osk $(A-C), b c d(D, E)$, and grk $(F)$ RNA in ovaries from $D f(2 L) T w 2 / D C O^{B 3}(A, C, E, F)$ or sibling $(B, D)$ females. The oocyte is at the posterior (right). $(A)$ Early stages of osk RNA localization are not disrupted by PKA mutations. osk RNA accumulates in the oocyte, beginning in the germarium (Ephrussi et al. 1991; Kim-Ha et al. 1991). (B) osk RNA is localized to the posterior of the oocyte in stage 9 egg chambers from control females. (C) At stage 9, osk has failed to reach the posterior in half of all PKA mutant oocytes. In most cases, accumulation near the middle of the oocyte is observed. (D) bcd RNA is localized to the anterior margin in control stage 8 oocytes. $(E)$ In oocytes from PKA mutant females, we see anterior localization of $b c d$ RNA as well as ectopic $b c d$ RNA in the posterior of many stage 8 oocytes. Posteriorly localized $b c d$ RNA can no longer be detected after stage 9 (not shown). (F) grk is localized normally in the anterior dorsal region of stage 8 oocytes from PKA mutant females.

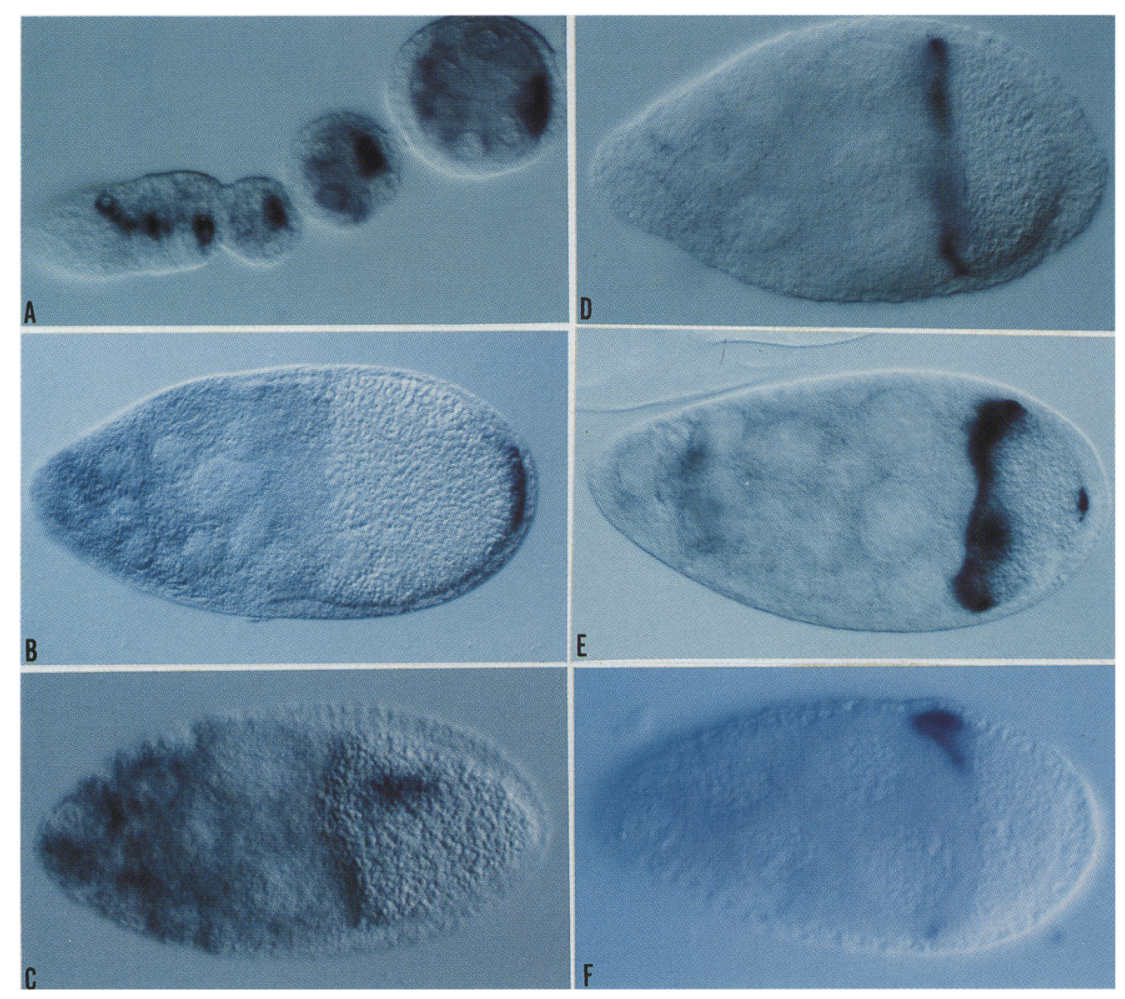

to the anterior and then becomes concentrated at the anterior-dorsal margin of the oocyte during stage 7 and early stage 8 . We did not detect any differences in the localization of grk RNA between oocytes from wild-type and PKA-deficient females (Fig. 1F). Furthermore, chorions of mature PKA-deficient eggs showed normal dorsoventral patterning. This argues that the disruptions of osk and bcd localization caused by PKA mutations reflect a specific requirement for PKA function in the specification of the anteroposterior, but not the dorsoventral, axis.

\section{PKA mutations disrupt the localization of a kinesin fusion protein}

To investigate the role of microtubules in polarized localization of morphogenetic determinants in oogenesis, Clark et al. (1994) have constructed a kinesin:lacZ fusion gene (khc:lacZ) to act as a reporter of microtubule polarity. Kinesin has been shown to act as a plus-enddirected motor in vitro (Vale et al. 1985). In otherwise wild-type flies that express the khc:lacZ gene under the control of a promoter that is active in oogenesis, Kine$\sin : \beta$-gal is first detected in the oocyte early in stage 8 (Clark et al. 1994). The fusion protein is detected at progressively more posterior positions throughout stage 8 , and by early stage 9 it is tightly localized to the extreme posterior (Clark et al. 1994; Fig 2A). This is consistent with the expectation that the fusion protein would move from minus ends of microtubules, nucleated at the an- terior, toward the plus ends extending to the posterior (Theurkauf et al. 1992).

We used this reporter construct as an indirect assay of the effect of PKA mutations on microtubule polarity. In oocytes from sibling control females, Kinesin: $\beta$-gal could be detected in the oocyte at stage 8 , and all stage 9 oocytes showed localization to the posterior cortex, as was reported by Clark et al. (1994) for wild-type oocytes. However, in egg chambers from PKA-deficient females, we saw a failure to localize Kinesin: $\beta$-gal to the extreme posterior in $52 \%(25 / 48)$ of all stage 9 egg chambers. Instead, we saw Kinesin: $\beta$-gal protein, somewhat diffusely distributed, confined to an area that is $\sim 25 \%$ of the oocyte's length from the posterior (Fig. 2B). No obvious differences between mutant and wild-type egg chambers were detected before this stage.

We stained egg chambers with rhodamine-phalloidin to determine whether or not there was a correlation between the localization phenotype and a previously described defect in the integrity of nurse cell junctions associated with female sterile alleles of PKA (Lane and Kalderon 1993). The egg chamber in Figure 2B appears to have undergone a number of nurse cell fusions, as evidenced by the large area outlined by phalloidin in the top of the picture and the actin inclusions in the center of this area. We believe the actin inclusions are ring canals that have become detached from cell membranes and subsequently degenerated. Figure $2 \mathrm{C}$ shows an egg chamber in which nurse cell junctions appear relatively intact, yet Kinesin: $\beta$-gal failed to reach the posterior. 

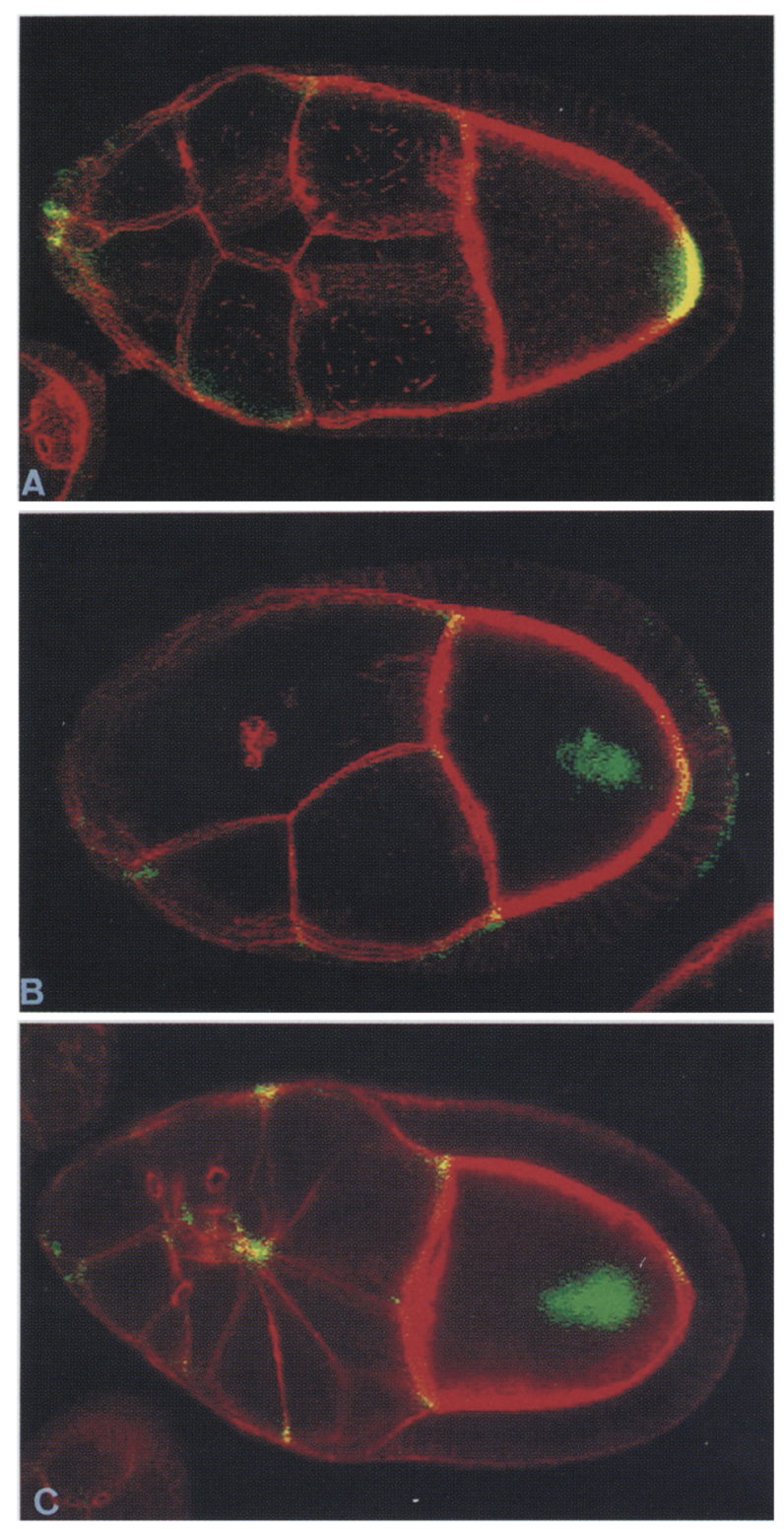

Figure 2. Kinesin fusion protein fails to accumulate at the posterior of PKA mutant oocytes. Egg chambers from PKA mutant and control sibling flies carrying a $\mathrm{P}$ element expressing a $\mathrm{Ki}$ nesin: $\beta$-gal fusion protein (Clark et al. 1994), double stained with rhodamine-phalloidin (red) and anti- $\beta$-galactosidase antibodies (green). (A) A stage 9 control oocyte. Kinesin: $\beta$-gal is tightly localized to the posterior cortex of the oocyte. $(B)$ A stage 9 oocyte from a PKA-deficient female. Kinesin: $\beta$-gal has not reached the posterior cortex and is somewhat more diffuse than in wild-type oocytes. In half of all mutant stage 9 oocytes, normal localization is seen (data not shown). (C) A stage 9 egg chamber from a PKA-deficient female. Phalloidin stains the cortical actin cytoskeleton underlying nurse cell and oocyte membranes and reveals relatively normal nurse cell morphology (cf. $B$ ). There does not appear to be a correlation between nurse cell integrity and Kinesin: $\beta$-gal localization.

Conversely, we saw egg chambers from mutant females that showed normal localization of Kinesin: $\beta$-gal but that had undergone many nurse cell fusions (data not shown|. Therefore, we do not think that the localization defects result form defects in nurse cell integrity but, rather, that these two phenotypes reflect two separate requirements for PKA in oogenesis.

\section{PKA activity is required in the germ line for Kinesin: $\beta$-gal localization}

Conditional alleles of the neurogenic genes $N$ and $D l$ result in patterns of localization for Kinesin: $\beta$-gal protein, $b c d$ RNA, and osk RNA similar to those in oocytes from females carrying PKA mutations (Ruohola et al. 1991; Clark et al. 1994). $N$ and DI appear to play several roles in oogenesis, particularly in the specification of follicle cell fate. Normally, the posterior polar follicle cells, a pair of follicle cells that are adjacent to the posterior of the oocyte, express the adhesion protein fasciclin III (Fas III) throughout most of oogenesis. In $N$ and $D I$ mutations, the number of posteriorly located follicle cells that express Fas III is between 5 and 20 (Ruohola et al. 1991). As both $N$ and $D l$ activities are required somatically for proper bcd and osk localization, it is thought that the mislocalization phenotypes result from the failure to specify correctly the fate of either the posterior polar follicle cells or their immediate neighbors. These cells have therefore been implicated in a signal transduction process by which positional information is communicated to the oocyte.

We asked whether PKA was required in the follicle cells, where it might play a role in the generation of a signal, or in the oocyte, where it might be activated in response to a signal. In PKA-deficient egg chambers, we observed normal Fas III staining in follicle cells, indicating that hypomorphic PKA mutations disrupt RNA localization without detectable alterations in follicle cell fates (data not shown). We also made use of an autosomal insertion of a $\mathrm{P}$ element carrying the $O \mathrm{vo}^{D 1}$ mutation (Chou et al. 1993), a dominant female sterile mutation, to generate germ-line clones homozygous for PKA null mutations. Germ-line recombinants were easily identified by the presence of a large ovary with vitellogenic eggs. Clones of the null alleles, $D C O^{B 3}$ and $D C O^{H 2}$, were recovered at similar frequencies to the parent nonmutagenized chromosome $(4-8 \%)$. These ovaries were dissected and stained with phalloidin and anti- $\beta$-galactosidase antibody. Many of these egg chambers showed numerous nurse cell fusions, but the geometry of the egg chamber was largely unaffected so that the localization of Kinesin: $\beta$-gal could be accurately scored. We observed a failure of Kinesin: $\beta$-gal to localize to the posterior of all 40 stage 9 oocytes from 26 females (Fig. 3), indicating that PKA activity is required in the germ line and that it is essential for the posterior localization of Kinesin: $\beta$ gal. Furthermore, normal Fas III staining was observed in polar follicle cells of these egg chambers, demonstrating that polar follicle cell specification does not depend on PKA activity in the germ line. 


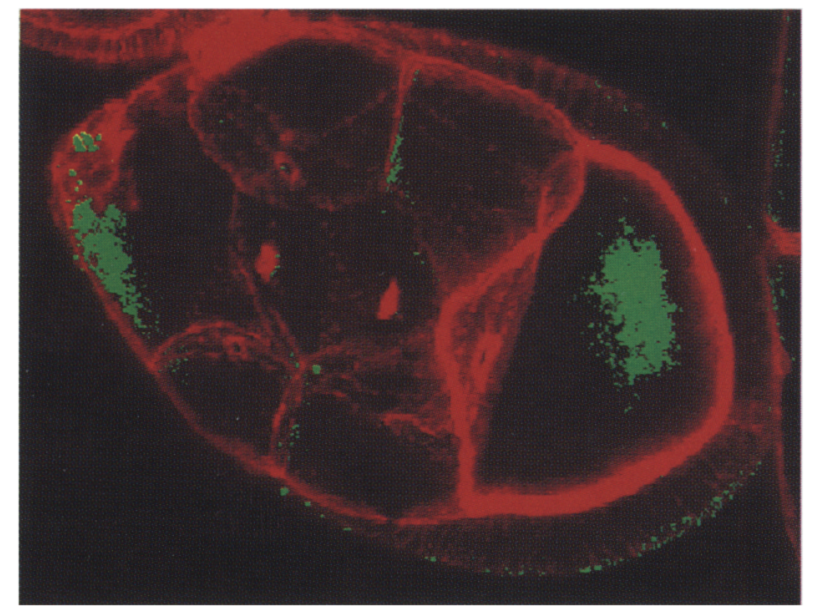

Figure 3. PKA is required in the germ line for Kinesin: $\beta$-gal localization. A stage 9 egg chamber, stained with rhodaminephalloidin (red) and anti- $\beta$-gal antibodies (green), from a clone of germ cells homozygous for the null allele $\mathrm{DCO}^{\mathrm{B} 3}$, induced in a $\mathrm{P}\left[\mathrm{ovo}^{D 1}\right] / D C 0^{B 3}$ female. In all 40 stage 9 oocytes from $26 \mathrm{fe}$ males, we saw a failure of Kinesin: $\beta$-gal to localize to the posterior of the oocyte. Some Kinesin: $\beta$-gal is seen in the nurse cells in both this and wild-type egg chambers at this stage.

\section{PKA mutations affect the distribution of microtubules in the oocyte}

For a more direct investigation of the relationship be- tween microtubule distribution and RNA localization, we compared the distribution of microtubules, revealed by anti-tubulin immunofluorescence, between wild-type and PKA-deficient oocytes. Theurkauf et al. (1992) have shown that in early egg chambers, the highest density of microtubules is seen in the oocyte. These microtubules originate from an MTOC that can be found in the posterior of a stage 6 oocyte. By stage 7, microtubules can no longer be seen at the posterior; instead the anterior margin of the oocyte acts as a nucleating region, and microtubules nucleated here continue to extend more posteriorly throughout stages 8-10a. During these stages, an anterior to posterior gradient of decreasing microtubule density can be seen.

In early egg chambers (stages 1-6), our observations are consistent with those of Theurkauf et al. $(1992,1993)$ and we did not detect any differences in microtubule distribution between wild-type and oocytes from PKA hypomorphs (Fig. 4A,D). However, at the time when bcd and osk RNAs are normally in the process of being localized to the anterior and posterior poles istages 7 and 8 ), clear differences were apparent. In $46 \%$ of mutant oocytes $(21 / 45)$ (Fig. 4E,F), but never in wild-type oocytes (Fig. 4B), microtubules were present at the posterior, in addition to the normal microtubule distribution at the anterior. In many cases $(11 / 21)$, the resolution was sufficient to show these as distinct populations of microtubules, consistent with their being nucleated from separate sites (Fig. 4F). Later in stage 9, microtubules extend

Figure 4. PKA mutations alter oocyte microtubule distribution. Antibodies to $\alpha$-tubulin reveal the distribution of microtubules in egg chambers. The follicle cells stain heavily for microtubules; in the germ line, the highest density is seen in the oocyte. Our results for wild-type microtubule distribution are generally in agreement with those described by Theurkauf et al. (1992). We focus on stage 6, prior to the reorganization of nucleation sites from posterior to anterior (Theurkauf et al. 1992), and early stage 8 , just after the oocyte nucleus has migrated to the dorsalanterior margin, as this is the earliest time when patterns of RNA localization in PKA-deficient females clearly differ from wild type. Only part of the egg chamber is shown as illustrated in $C$, with the oocyte
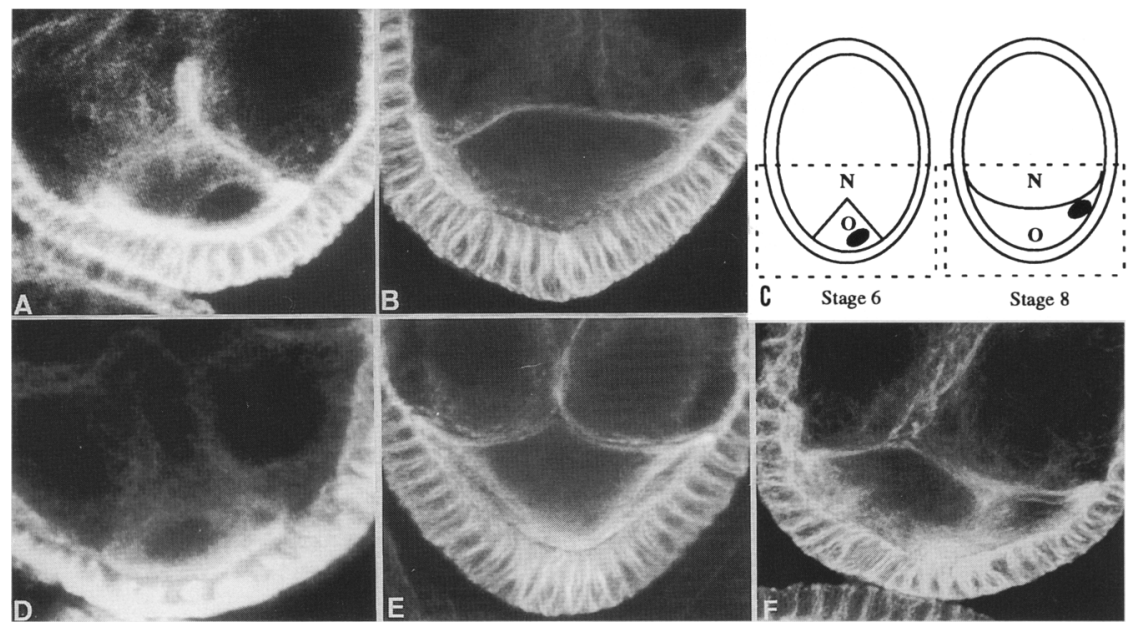

$(\mathrm{O})$ at the bottom, below the nurse cells $(\mathrm{N})$, and the oocyte nucleus indicated by a solid oval. $(A)$ Stage 6 egg chamber from wild-type female. Microtubules are concentrated in the oocyte, with highest levels at the posterior. $(B \mid$ Microtubule distribution in wild-type early stage 8 egg chamber. Intense staining is observed near the anterior margin of the oocyte and extending posteriorly along the cortex. The extreme posterior of the oocyte is free of microtubules. $(D)$ Stage 6 egg chamber from PKA-deficient female. Microtubule distribution is similar to that in wild-type egg chambers at this and earlier stages. $(E)$ Stage 8 egg chamber from a mutant female. Microtubules are seen all along the oocyte cortex, including a high density at the posterior. $(F)$ In many egg chambers from mutant females with increased microtubule density at the posterior, the posterior microtubules appear to have a separate origin from those at the anterior, with a point of maximum intensity at the extreme posterior. This is consistent with separate sites of nucleation for the anterior and posterior microtubule populations. Tubulin staining following partial depolymerization of microtubules reveals a microtubule nucleating site at the extreme posterior of stage 6 wild-type oocytes that is no longer present at stage 7 (Theurkauf et al. 1992). In similar experiments, tubulin staining was seen at the extreme posterior of stage 7 oocytes from PKA hypomorphs $(7$ of 20 showing good staining) but never in stage 7 oocytes from control siblings (0 of 50). These data support the inference that ectopic microtubules at the posterior of PKA mutant stage 7 oocytes $|E, F|$ are nucleated at the posterior. 
farther along the cortex toward the posterior in wild-type oocytes. Thus, although posterior microtubules were present in many mutant oocytes at this stage, they were not obviously discontinuous with microtubules extending from the anterior. We cannot, therefore, determine how long the microtubules found at the posterior of stage 7 and 8 mutant oocytes persist. We do conclude, however, that an abnormal microtubule distribution is present in mutant oocytes, at the time when $b c d$ and osk RNAs are normally transported to the anterior and posterior of the oocyte (Fig. 5).

\section{Discussion}

In this study we have shown that PKA mutations affect the localization of RNAs along the anteroposterior axis of the Drosophila oocyte. Direct visualization of microtubules has shown that PKA mutations also affect the reorganization of the microtubule cytoskeleton that normally occurs immediately prior to polarized RNA localization. We argue that these two effects are causally related and that PKA is absolutely required in the oocyte to transduce a temporal signal from posterior follicle cells that initiates microtubule reorganization and, hence, the correct polar localization of RNAs.

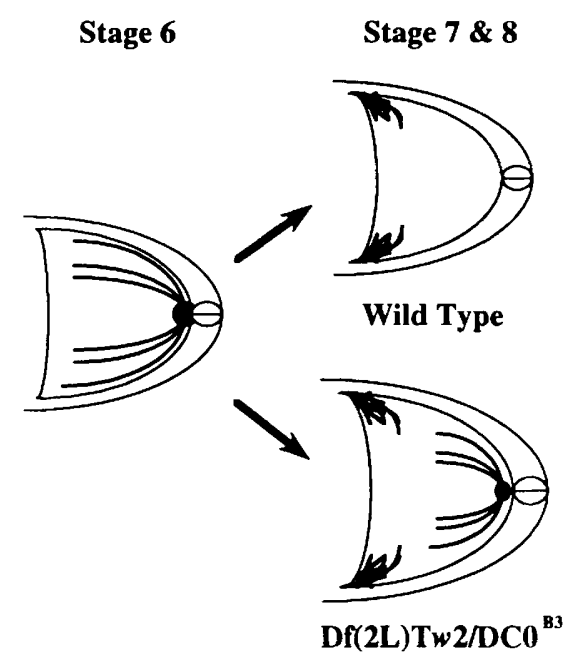

Figure 5. Diagrammatic representation of microtubule distribution in wild-type and PKA mutant oocytes. In early egg chambers, high microtubule density is observed in the oocyte, and partial depolymerization experiments suggest that an MTOC is present at the posterior of stage 6 oocytes. This population is no longer detected at stage 7 , and new microtubules are present at the anterior margin of the oocyte, which appears to act as a nucleating region (Theurkauf et al. 1992). In oocytes from PKA mutant females, which have apparently normal microtubule distribution up to stage 6 , the population of microtubules at the posterior of the oocyte persists until stages 7 and 8 , while microtubules are being nucleated at the anterior. According to the current models for microtubule polarity in oocytes, this would result in microtubule minus ends being located at the anterior and posterior poles (as opposed to only the anterior in wild type), whereas plus ends, found at the posterior of wild-type oocytes, would be found near the middle of the oocyte.

\section{Specificity of PKA action in oogenesis}

Intensive investigation of the effects of PKA mutations on oogenesis has revealed two specific and distinct phenotypes, the concomitant mislocalization of RNAs and microtubules at stages 7 and 8 and the progressive fusion of nurse cells during stages 5-10. Even germ-line null mutations of the catalytic subunit gene have no additional effects, allowing the formation of a mature oocyte and normal nurse cell-oocyte transport (Lane and Kalderon 1994), a process that requires an intact filamentous actin cytoskeleton (Knowles and Cooley 1994). We believe that the requirement for PKA in oocyte polarity is distinct from its role in nurse cell junctional integrity for several reasons. First, we did not see a correlation between the severity of these two incompletely penetrant phenotypes in individual egg chambers of PKA hypomorphs. Second, germ-line armadillo mutations cause extensive fusion of nurse cells without affecting RNA localization (Peifer et al. 1993). Third, the association of catalytic subunit protein with nurse cell membranes (Lane and Kalderon 1994) suggests that it has access to proteins associated with the membrane skeleton and therefore is likely to play a very direct role in the stabilization of cell junctions. In contrast, the disruptions of microtubules, RNA, and Kinesin: $\beta$-gal localization that we observed were confined to the posterior of the oocyte, far removed from nurse cell junctions. The temporal and spatial selectivity of PKA mutations on RNA localization further emphasizes the specificity of its action in the oocyte. Thus, the initial oocyte-specific accumulation of osk RNA and the concentration of microtubules at the oocyte posterior prior to stage 7 were unaffected by PKA mutations, as were the anterior accumulation of $b c d$ RNA and the dorsal anterior localization of $g r k$ RNA, which normally accompany the posterior localization of osk RNA at stage 8 .

\section{PKA and microtubule reorganization}

The distribution of microtubules in egg chambers has been shown to be dynamic, with distinct phases that correlate with two phases of RNA accumulation in the oocyte. In the germarial stages, a single microtubule cytoskeleton forms within the 16-cell cyst originating from an MTOC in the oocyte (Theurkauf et al. 1993). Partial depolymerization experiments demonstrate the presence of this MTOC until stage 6, when it is found at the posterior of the oocyte. The loss of this MTOC and the appearance of microtubule nucleation sites at the anterior is seen during and after stage 7 (Theurkauf et al. 1992). Thus, in wild-type egg chambers microtubules nucleated at the posterior are lost prior to the appearance of microtubules nucleated at the anterior. In PKA mutants a high density of microtubules was observed at the posterior of stage 7 and 8 oocytes, well after microtubules had formed at the anterior. The logical assumption that these ectopic microtubules were persisting from previous stages and, therefore, nucleated at the posterior is supported by three observations. First, these microtu- 
bules were often seen to be clearly discontinuous with microtubules extending from the anterior. Second, preliminary evidence from partial depolymerization experiments shows the abnormal persistence of an MTOC at the extreme posterior of stage 8 oocytes from PKA hypomorphs (see legend to Fig. 4). Third, as discussed below, the localization of $b c d$ and osk RNAs, as well as the kinesin fusion protein, was consistent with the inferred polarity of microtubules that would result from persistence of microtubules nucleated at the posterior.

The appearance of microtubules at the anterior margin proceeded normally in PKA mutants and, therefore, appears to be independent of the depolymerization of microtubules at the posterior. It is possible that molecules that are capable of nucleating microtubules are supplied by the nurse cells during stage 7 . The location of the anterior margin of the oocyte adjacent to the nurse cells could lead to the preferential accumulation of microtubule-nucleating molecules at the point of entry. The distribution of molecules such as $\gamma$-tubulin (Stearns and Kirchner 1994) and pericentrin (Doxsey et al. 1994) will be particularly informative in addressing this issue.

\section{RNA localization and microtubule organization}

A number of recent studies have provided evidence of a link between microtubules and localization of $b c d$ and osk RNAs. The use of drugs that depolymerize microtubules can disrupt both the initial oocyte specific and later polarized accumulation of osk and bcd RNAs (Pokrywka and Stephenson 1991; Theurkauf et al. 1993; Clark et al. 1994). Also, exu protein, which is required for $b c d$ RNA localization, has been found in large particles that colocalize with microtubules (Wang and Hazelrigg 1994).

Directional microtubule-based transport requires an appropriate geometry of microtubules, signals on the cargo to specify destination and transporters that both recognize destination tags and actively translocate along microtubules. PKA mutations cannot be affecting the cargo itself, as transport of several macromolecules was affected, each of which was present in its normal location prior to transport. It is possible that PKA regulates the activity of motor proteins or their engagement with cargo and microtubules, as has been suggested for the movement of organelles in neurons (Azhderian et al. 1994). However, this would not easily explain the normal accumulation of $b c d$ and grk RNA at the anterior nor the ectopic accumulation of $b c d$ RNA at the posterior. In contrast, the third possibility, that PKA affects RNA localization by regulating the distribution of $\mathrm{mi}$ crotubules, is supported by several observations. Most important, we saw clear defects in microtubule distribution in PKA hypomorphs at the time when RNA localization and active microtubule reorganization were in progress. Second, the penetrance of microtubule and RNA localization defects in PKA hypomorphs were very similar. Finally, the aberrant localization of Kinesin: $\beta$ gal, which was designed to report the distribution of microtubules and has been found empirically to colocalize with osk RNA in a variety of mutant backgrounds (this paper; Clark et al. 1994), was consistent with the penetrance of both phenotypes and with a coherent explanation of how microtubule polarity prefigures RNA localization in the oocyte.

In the absence of either formal demonstrations of $\mathrm{mi}$ crotubule polarity or the immunolocalization of molecules known to be required for microtubule nucleation, it is not possible to establish indisputably the relation ship between microtubule polarity and the anteroposterior axis of the oocyte. However, the implication of the observations by Theurkauf et al. (1992) is that the position of minus ends of microtubules shifts from posterior to anterior between stages 6 and 7, prior to the time when $b c d$ and osk RNAs are first seen to acquire polarized distribution (Fig. 5). This would allow for the initial accumulation of RNAs in the oocyte by association with minus-end-directed motors. Subsequent localization of $b c d$ to the anterior would also be facilitated by association with minus-end-directed motors, whereas localization of osk to the posterior of stage 8-9 oocytes would require plus-end-directed motors (Theurkauf et al. 1992). The similarity between the distribution of Kinesin: $\beta$-gal and osk RNA in wild-type oocytes, as well as oocytes form $N, D l$, capu, and spir mutant females, is highly suggestive of plus-end-directed transport of osk RNA (Clark et al. 1994).

On the basis of these models, the transient ectopic posterior localization of bcd RNA in PKA-deficient oocytes can be explained by the persistence of microtubule minus ends at the posterior of oocytes into stages 7 and 8 (Fig. 5). Posterior $b c d$ RNA may be transient either if minus ends are lost from the posterior after stage 8 or if factors required to anchor $b c d$ RNA to the cytoskeleton are absent from the posterior of the oocyte. Similarly, these microtubules may serve to direct osk and Kinesin: $\beta$-gal away from their minus ends at the posterior toward the center of the oocyte. Colocalization of stau protein with osk RNA has been observed in all mutant cases examined (St. Johnston et al. 1991; Clark et al. 1994) and would account for the persistence of ectopic osk RNA in PKA mutant oocytes at stages 9 and 10.

\section{Signal transduction and oocyte polarity}

Most genetic studies on the specification of the anteroposterior axis have led to the proposal of models for a germ-line autonomous mechanism for the generation of polarity, in contrast with the acknowledged role for communication between germ-line and somatic cells in the generation of dorsoventral polarity and in terminal specification (Stein and Stevens 1991). However, Ruohola et al. (1991) showed that genes required for follicle cell specification can have an effect on anteroposterior axis formation and proposed a model whereby follicle cells situated adjacent to the oocyte posterior communicate information to the oocyte. Because $N$ and $D l$ mutations affect the specification of posterior follicle cells, leading to hyperptrophy of cells expressing Fas III, it is possible that the observed phenotype reflects a neomorphic con- 
dition, brought about by the generation of a signaling pathway that is never used in normal oogenesis. We have now observed a similar RNA localization phenotype resulting from mutations in the catalytic subunit of PKA that we have shown to be recessive loss-of-function alleles by biochemical and phenotypic assays (Lane and Kalderon 1993). Furthermore, we have shown that PKA activity is required in the germ line, whereas large follicle cell clones that are null for catalytic subunit have no effect on localization of Kinesin: $\beta$-gal (M.E. Lane, unpubl.). These observations support the model of Ruohola et al. (1991) (see also Clark et al. 1994; Ruohola-Baker et al. 1994) and suggest a molecular mechanism for the generation of anteroposterior polarity in the oocyte.

A polarized microtubule cytoskeleton exists in egg chambers from stages 1-10a. However, the polarity of the microtubule cytoskeleton changes between stage 6 and 7. In early previtellogenic stages, the microtubule organization facilitates unidirectional transport of materials into the transcriptionally latent oocyte. In later stages as the oocyte grows by yolk uptake, cytoplasmic determinants must be distributed asymmetrically for the specification of the embryonic axes. We have presented evidence that the reorganization of microtubules between stages 6 and 7 is essential for localization of macromolecules at the posterior of the oocyte. We suggest that the redistribution of microtubules is governed by a signal, emanating from either the posterior polar follicle cells or their immediate neighbors, the flanking cells, which results in the activation of PKA, presumably via an elevation in cAMP concentration. In PKA hypomorphs, approximately half of stage 7-8 egg chambers showed abnormal persistence of microtubules at the posterior, ectopic $b c d$ RNA at the posterior, and a failure to localize osk RNA or Kinesin: $\beta$-gal to the posterior. In contrast, null mutations of the PKA catalytic subunit, $D C O$, in the germ line prevented posterior localization of Kinesin: $\beta$-gal in all egg chambers, implying that PKA is essential for signal transduction and that the partially penetrant phenotypes of hypomorphs were attributable to residual PKA activity rather than the use of an alternative signal transduction pathway.

We are interested in determining the identity of targets of PKA phosphorylation in this process, as we believe that they will provide information about the control of microtubule dynamics. The microtubule-associated protein (MAP2) has been shown to be a major target of PKA phosphorylation in extracts from vertebrate brain tissue (Sloboda et al. 1975; Theurkauf and Vallee 1983), and type-II PKA has been found to be associated with MAP2 in these extracts (Theurkauf and Vallee 1982). Association of MAP2 with microtubules can inhibit depolymerization of microtubules, and PKA phosphorylation of MAP2 can release this inhibition (Jameson and Caplow 1981). A mechanism involving MAP2 or a MAP2like factor for microtubule redistribution in the oocyte would be consistent with our observations. Alternatively, PKA targets might include centrosomal proteins that regulate the nucleation activity of the posterior MTOC. The type-II regulatory subunit of PKA localizes to centrosomes in cultured neuronal, glial, epithelial, and fibroblastic cells (Nigg et al. 1985; De Camilli et al. 1986). In addition, a number of centrosomal antigens have been identified that show cell cycle-specific phosphorylation states, suggesting that changes in the nucleating activity of centrosomes are regulated by phosphorylation (Centonze and Borisy 1990; Ohta et al. 1993). It is possible, therefore, that the activation of PKA in the oocyte is required to inhibit nucleation activity of the posterior MTOC during and after stage 6 , and that the presence of active nucleation sites at the anterior results in net depolymerization of microtubules associated with the inactivated posterior MTOC. In this mode of action the effects of PKA could be spatially limited both by the local generation of a cAMP signal at sites of contact with posterior follicle cells and by the anchoring of PKA in the region of the MTOC by cytoskeleton-associated proteins that bind to the regulatory subunit of PKA.

\section{Materials and methods}

Drosophila stocks

For in situ hybridizations and tubulin immunofluorescence, ovaries from $D C 0^{B 3} / D f(2 L) T w 2 ; r y^{506}$ were analyzed. Sibling females from the same cross, which were either $D C 0^{B 3} / C y o$ or $D f(2 L) T w 2 / C y O$, were pooled and served as internal controls. In all cases, control ovaries reproducibly showed wild-type patterns of RNA localization and microtubule distribution.

Flies bearing the khc:lacZ fusion gene on an enhancer trap transposon inserted on the third chromosome, designated KZ503, were provided by Ira Clark and described in Clark et al. (1994). $D C 0^{B 3} / D f(2 L) T w 2$ females, which were heterozygous for KZ503, were analyzed for Kinesin: $\beta$-gal localization by antibody staining (described below), with siblings as controls.

\section{Isolation of ovaries}

Ovaries from 50-500 females were isolated and dissociated into individual egg chambers as described by Theurkauf et al. (1992). Alternatively, ovaries were dissected by hand in cold modified Robb's medium.

\section{In situ hybridization}

Hybridization was performed essentially according to Tautz and Pfeifle (1989) with modifications suggested by Liz Gavis and Chris Rongo (Whitehead Institute, Cambridge, MA). Approximately $20 \mu \mathrm{l}$ of ovaries (mass isolated, or dissected from 10 females) was fixed in $4 \%$ paraformaldehyde, $10 \% \mathrm{DMSO}$, and $10 \%$ bleach for $30 \mathrm{~min}$ with gentle rotation, followed by $5 \times 5$ min washes in PBS, $1 \%$ Tween 20 (PBT). Proteinase K conditions varied for each probe generally, best results were obtained with a $15-\mathrm{min}$ treatment and proteinase concentration between $20-100 \mu \mathrm{g} / \mathrm{ml}$ in PBT when riboprobes were used (grk). Onehour treatments with $100 \mu \mathrm{g} / \mathrm{ml}$ of proteinase $\mathrm{K}$ gave best results when DNA probes were used $|b c d, o s k|$. Ovaries were rinsed twice and then washed $2 \times 5 \mathrm{~min}$ in PBT. After postfixation for $20 \mathrm{~min}$ in $4 \%$ paraformaldehyde, ovaries were washed five times in PBT and stored frozen in 9:1 methanol/DMSO or hybridized according to Tautz and Pfeifle (1989). All subsequent steps were according to Tautz and Pfeifle (1989). Ovaries were mounted in Aquaploymount (Polyscience).

Riboprobes were synthesized from cDNAs subcloned into 
Bluescript Vectors using the Boehringer RNA labeling kit and instructions provided. Following the transcription reaction, probes were hydrolyzed in carbonate buffer $(2 \times: 120 \mathrm{mM}$ $\mathrm{Na}_{2} \mathrm{CO}_{3}, 80 \mathrm{~mm} \mathrm{NaHCO}$ at $\mathrm{pH} \mathrm{10.2)} \mathrm{for} 40 \mathrm{~min}$ at $65^{\circ} \mathrm{C}$. Following addition of $100 \mu \mathrm{l}$ of $0.2 \mathrm{M} \mathrm{NaOAc}(\mathrm{pH} 6)$ to stop the reaction, the probe was precipitated by adding $\mathrm{LiCl}$ to $0.4 \mathrm{M}, 20$ $\mu \mathrm{g}$ of tRNA and $600 \mu \mathrm{l}$ of ethanol. Following precipitation at $-20^{\circ} \mathrm{C}$, the pellet was resuspended in $150 \mu \mathrm{l}$ of hybridization buffer (Tautz and Peifle 1989), and $0.5 \mu \mathrm{l}$ of this stock was added to a $500-\mu l$ hybridization reaction. DNA probes were synthesized according to Tautz and Pfeifle (1989).

\section{Antibody staining}

Ovaries were either dissected or mass isolated. In general, ovaries were fixed in $4 \%$ paraformaldehyde for $30 \mathrm{~min}$, washed in PBT, and blocked for $1 \mathrm{hr}$ in PBSTT (PBS, $1 \%$ BSA, $0.1 \%$ Triton, $0.1 \%$ Tween). Primary antibodies were diluted in PBSTT and incubated overnight at $4^{\circ} \mathrm{C}$. Rabbit anti- $\beta$-galactosidase antibody (Cappel) was diluted 1:5000.

Ovaries were washed $4 \times 15 \mathrm{~min}$ in PBT, and incubated for 2 $\mathrm{hr}$ at room temperature with fluourescein or Texas Red-conjugated secondary antibodies (Cappel), which were preabsorbed against fixed ovaries and diluted 1:500, for $2 \mathrm{hr}$ at room temperature. After $4 \times 15$ min washes in PBT, ovaries were mounted in Aquaploymount (Polyscience).

Ovaries for microtubule staining were fixed for $10 \mathrm{~min}$ in $-20^{\circ} \mathrm{C}$ methanol, and washed and stained as above. A monoclonal antibody against $\alpha$-tubulin (Sigma) was used at 1:500. The degree to which oocyte microtubules could be detected varied, depending on the degree of microtubule preservation and antibody penetration. Data were collected from preparations in which the degree of microtubule preservation allowed for the visualization of mitotic spindles in the germarium and dividing follicle cells, and antibody penetration permitted readily visible microtubule staining at the oocyte anterior by conventional fluorescence microscopy. Confocal images were obtained from egg chambers between stages 7 and 8 , when the oocyte nucleus had completed its migration to the dorsal anterior margin. Eight to ten $1 \mu \mathrm{m}$ optical sections were obtained, and four of these sections were projected to produce the images presented in Figure 4. Samples were taken from three separate experiments, to give a total of 45-50. In all three experiments the proportion of mutants that showed posterior microtubule density was similar.

\section{Mosaic analysis}

To induce germ-line clones, first-instar larvae $(36-48 \mathrm{hr}$ old $)$ from the cross $y w ; D C 0^{x} / C y O ; \mathrm{KZ} 503 \times \mathrm{P}\left[0 v 0^{D 1}[/ C y O\right.$ were $\gamma$-irradiated at a constant dose of 1000 rads. $x$ indicates $D C O$ alleles $\mathrm{B} 3$ and $\mathrm{H} 2$, which are null alleles, and the parent nonmutagenized chromosome, S6 (Lane and Kalderon 1993), and $\mathrm{P}\left[\mathrm{ovo}^{D 1}\right]$ indicates the insertion $13 \times 13$ described by Chou et al. (1993). Chromosomes carrying $D C O^{B 3}$ and $D C O^{H 2}$ are free of other lethals, as both can be rescued by a $D C O$ genomic transposon (Lane and Kalderon 1993; M.E. Lane and D. Kalderon, unpubl.). Adult females of the genotype $D C O^{x} / \mathrm{P}\left[0 v 0^{D 1}\right]$ were collected, crossed with males, and kept in vials with wet yeast for 3-5 days. Ovaries from 100-200 females of each genotype were dissected. Clones of germ-line cells homozygous for $\mathrm{S} 6$ or DCO mutations could be identified easily by the presence of ovarioles with vitellogenic egg chambers, which are not produced by nonrecombinant females (Chou et al. 1993). Ovaries were stained with rhodamine isothiocyanate (RITC) phalloidin and anti- $\beta$-galactosidase antibodies as described above.

\section{Acknowledgments}

We are very grateful to Ira Clark for providing Kinesin: $\beta$-gal fly stocks and information prior to publication. We thank Dr. Norbert Perrimon for providing the autosomal $\mathrm{P}\left[\right.$ ovo $\left.^{D 1}\right]$ stock prior to publication; members of the Hazelrigg, Lehmann, and Schüpbach laboratories for providing probes for in situ hybridizations; Bill Theurkauf for advice about anti-tubulin immunofluorescence; Nik Necles for invaluable assistance with photography; Laura Wolszon for instruction with confocal microscopy; and Steve Mount, Tulle Hazelrigg, Bill Theurkauf, and Steve DiNardo for comments on the manuscript. This work was supported by a predoctoral training grant form the National Institutes of Health (NIH) to M.E.L. and NIH grant GM41815 and a Pew Scholars Award to D.K.

The publication costs of this article were defrayed in part by payment of page charges. This article must therefore be hereby marked "advertisement" in accordance with 18 USC section 1734 solely to indicate this fact.

\section{References}

Ainger, K., D. Avossa, F. Morgan, S.J. Hill, C. Barry, E. Barbarese, and J.H. Carson. 1993. Transport and localization of exogenous myelin basic protein mRNA microinjected into oligodendrocytes. I. Cell Biol. 123: 431-441.

Azhederian, E.M., D. Hefner, C.-H. Lin, L.K. Kaczmarek, and P. Forscher. 1994. Cyclic AMP modulates fast axonal transport by increasing the probability of single organelle movement. Neuron 12: 1223-1233.

Centonze, V.E. and G.G. Borisy. 1990. Nucleation of microtubules from mitotic centrosomes is modulated by a phosphorylated epitope. I. Cell Sci. 95: 405-411.

Chou, T.-B., E. Noll, and N. Perrimon. 1993. Autosomal $\mathrm{P}\left[\right.$ ovo $\left.^{\mathrm{D} 1}\right]$ dominant female-sterile insertions in Drosophila and their use in generating germline chimeras. Development 119: 1359-1369.

Clark, I., E. Giniger, H. Ruohola-Baker, L.Y. Jan, and Y.N. Jan. 1994. Transient posterior localization of a kinesin fusion protein reflects anteroposterior polarity of the Drosophila oocyte. Curr. Biol. 4: 289-300.

De Camilli, P., M. Moretti, S. Denis Donini, U. Walter, and S.M. Lohmann. 1986. Heterogeneous distribution of the cAMP receptor protein RII in the nervous system: Evidence for its intracellular accumulation on microtubules, microtubuleorganizing centers, and in the area of the Golgi complex. $I$. Cell Biol. 103: 189-203.

Doxsey, S.J., P. Stein, L. Evans, and M. Kirschner. 1994. Pericentrin, a highly conserved centrosome protein involved in microtubule organization. Cell 76: 639-650.

Ephrussi, A., L.K. Dickenson, and R. Lehmann. 1991. oskar organizes the germ plasm and directs localization of the posterior determinant nanos. Cell 66: 23-35.

Ferrandon, D. and D. St. Johnston. 1992. The localization of the maternal signals that determine the anterior-posterior axis of the Drosophila embryo. Sem. Dev. Biol. 3: 3-13.

Gavis, E.R. and R. Lehmann. 1992. Localization of nanos RNA controls embryonic polarity. Cell 71: 301-313.

Hay, B., L.Y. Jan, and Y.N. Jan. 1990. Localization of vasa, a component of Drosophila polar granules, in maternal effect mutants that alter embryonic anteroposterior polarity. Development 109: 425-433.

Hirokawa, N. 1993. Mechanism of axonal transport. Identification of new molecular motors and regulators of transport. Neurosci. Res. 18: 1-9.

Jameson, L. and M. Caplow. 1981. Modification of microtubule 
steady-state dynamics by phosphorylation of the microtubule-associated proteins. Proc. Natl. Acad. Sci. 78: 34133417 .

Kim-Ha, J., J.L. Smith, and P.M. Macdonald. 1991. oskar mRNA is localized to the posterior pole of the Drosophila oocyte. Cell 66: 23-35.

Kim-Ha, J., P.J. Webster, J.L. Smith, and P.M. Macdonald. 1993. Multiple RNA regulatory elements mediate distinct steps in localization of oskar mRNA. Development 119: 169-178.

Knowles, B.A. and L. Cooley. 1994. The specialized cytoskeleton of the Drosophila egg chamber. Trends Genet. 10: 235241.

Lane, M.E. and D. Kalderon. 1993. Genetic investigation of cAMP-dependent protein kinase function in Drosophila development. Genes \& Dev. 7: 1229-1243.

- 1994. Localization and functions of protein kinase A during Drosophila oogenesis. Mech. Dev. (in press).

Lasko, P.F. and M. Ashburner. 1990. Posterior localization of vasa protein correlates with, but is not sufficient for, pole cell development. Genes \& Dev. 4: 905-921.

Macdonald, P.M. 1992. The means to the ends: Localization of maternal messenger RNAs. Sem. Dev. Biol. 3: 413-424.

Macdonald, P.M., K. Kerr, J.L. Smith, and A. Leask. 1993. RNA regulatory element BLE1 directs the early steps of bicoid mRNA localization. Development 118: 1233-1243.

Mitchison, T.J. 1992. Compare and contrast actin filaments and microtubules. Mol. Biol. Cell 3: 1309-1315.

Mowry, K.L. and D.A. Melton. 1992. Vegetal messenger RNA localization directed by a 340-nt RNA sequence element in Xenopus oocytes. Science 255: 991-994.

Neuman-Silberberg, F.S. and T. Schüpbach. 1993. The Drosophila dorsoventral patterning gene gurken produces a dorsally localized RNA and encodes a TGF $\alpha$-like protein. Cell 75: 165-174.

Nigg, E.A., G. Schafer, H. Hilz and H.M. Eppenberger. 1985. Cyclic-AMP-dependent protein kinase type II is associated with the Golgi complex and with centrosomes. Cell 44: 1039-1051.

Ohta, K., N. Shiina, E. Okumura, S-I. Hisanaga, T. Kishimoto, S. Endo, Y. Gotoh, E. Nishida, and H. Sakai. 1993. Microtubule nucleating activity of centrosomes in cell-free extracts from Xenopus eggs: Involvement of phosphorylation and accumulation of pericentriolar material. I. Cell Sci. 104: 125-137.

Peifer, M., S. Orsulic, D. Sweeton, and E. Wieschaus. 1993. A role for the Drosophila segment polarity gene armadillo in cell adhesion and cytoskeletal integrity during oogenesis. Development 118: 1191-1207.

Pokrywka, N.J. and E.C. Stephenson. 1991. Microtubules mediate the localization of bicoid RNA during Drosophila oogenesis. Development 113: 55-66.

Ruohola, H., K.A. Bremer, D. Baker, J.R. Swedlow, L.Y. Jan, and Y.N. Jan. 1991. Role of neurogenic genes in establishment of follicle cell fate and oocyte polarity during oogenesis in Drosophila. Cell 66: 433-449.

Ruohola-Baker, H., L.Y. Jan, and Y.N. Jan. 1994. The role of gene cassettes in axis formation during Drosophila oogenesis. Trends Genet. 10: 89-94.

Sloboda, R.D., S.A. Rudolf, J.L. Rosenbaum, and P. Greengard. 1975. Cyclic AMP dependent endogenous phosphorylation of a microtubule-associated protein. Proc. Natl. Acad. Sci. 72: $177-181$.

St. Johnston, D., W. Driever, T. Berleth, S. Richstein, and C. Nüsslein-Volhard. 1989. Multiple steps in the localization of bicoid RNA to the anterior pole of the Drosophila oocyte. (Suppl.) Development 107: 13-19.

St. Johnston, D., D. Beuchle, and C. Nüsslein-Volhard. 1991. staufen, a gene required to localize maternal RNAs in the Drosophila egg. Cell 66: 51-63.

Stearns, T. and M. Kirschner. 1994. In vitro reconstitution of centrosome assembly and function: The central role of $\gamma$-tubulin. Cell 76: 623-637.

Stein, D.S. and L.M. Stevens. 1991. Establishment of the dorsalventral and terminal pattern in the Drosophila embryo. Curr. Opin. Genet. Dev. 1: 247-254.

Tautz, D. and C. Pfeifle. 1989. A nonradioactive in situ hybridization method for the localization of specific RNAs in the Drosophila embryos reveals a translational control of the segmentation gene hunchback. Chromosoma 98: 81-85.

Theurkauf, W.E. and R.B. Vallee. 1982. Molecular characterization of the cAMP-dependent protein kinase bound to microtubule associated protein 2. I. Biol. Chem. 257: 3284-3290.

-1983. Extensive cAMP dependent and cAMP independent phosphorylation of microtubule associated protein $2 . I$. Biol. Chem. 258: 7883-7886.

Theurkauf, W.E., S. Smiley, M.L. Wong, and B.M. Alberts. 1992. Reorganization of the cytoskeleton during Drosophila oogenesis: Implication for axis specification and intercellular transport. Development 115: 923-936.

Theurkauf, W.E., B.M. Alberts, Y.N. Jan, and T.A. Jongens. 1993. A central role for microtubules in the differentiation of Drosophila oocytes. Development 118: 1169-1180.

Vale, R.D., B.J. Schnapp, T. Mitchison, E. Steuer, T.S. Reese, and M. Sheetz. 1985. Different axioplasmic proteins generate movement in opposite directions along microtubules in vitro. Cell 43: 623-632.

Wang, S. and T. Hazelrigg. 1994. Implications for bcd mRNA localization from spatial distribution of exu protein in Drosophila oogenesis. Nature 369: 400-403.

Wilhelm, R.J. and R.D. Vale. 1993. RNA on the move: The mRNA localization pathway. I. Cell Biol. 123: 269-274.

Yisraeli, J.K., S. Sokol, and D.A. Melton. 1990. A two-step model for the localization of maternal mRNA in Xenopus oocytes: Involvement of microtubules and microfilaments in the translocation and anchoring of $\mathrm{Vgl} \mathrm{mRNA.} \mathrm{Develop-}$ ment 108: 289-298. 


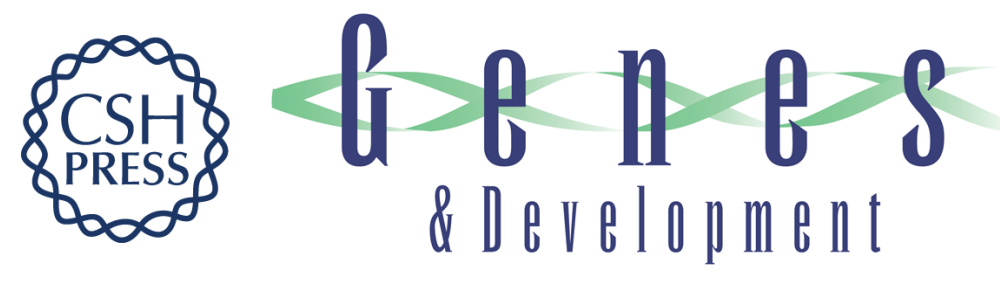

\section{RNA localization along the anteroposterior axis of the Drosophila oocyte requires PKA-mediated signal transduction to direct normal microtubule organization.}

M E Lane and D Kalderon

Genes Dev. 1994, 8:

Access the most recent version at doi:10.1101/gad.8.24.2986

References This article cites 43 articles, 23 of which can be accessed free at:

http://genesdev.cshlp.org/content/8/24/2986.full.html\#ref-list-1

License

Email Alerting Receive free email alerts when new articles cite this article - sign up in the box at the top Service right corner of the article or click here.

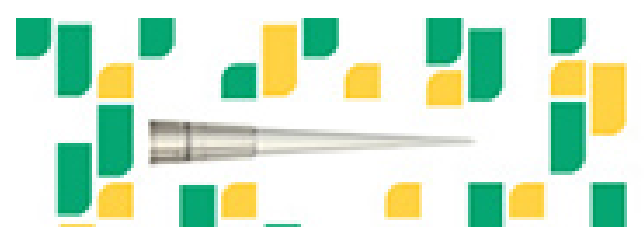

Focused on your science. 